\title{
Onset of Marangoni convection in a saturated porous medium
}

\begin{abstract}
The onset of Marangoni convective instabilities in a porous layer is studied by means of linear stability analysis. The upper and lower boundaries of the porous layer are fixed with a constant heat flux. The Brinkman model is used and the Darcy law is employed to describe the flow in the porous medium heated from below. The asymptotic solutions of long wavelength are derived analytically by using regular perturbation technique.
\end{abstract}

Keyword: Linear stability; Marangoni convection; Porous medium 